\title{
An element of a finite monoid is right invertible if and only if it is left invertible
}

\author{
Open Mathematics Collaboration*†
}

August 7, 2020

\begin{abstract}
We prove the proposition addressed in the title of this paper.
\end{abstract}

keywords: finite monoid, left invertible, right invertible, abstract algebra

The most updated version of this paper is available at https://osf.io/7vm92/download

\section{Notation \& Definition}

1. $[1,2]$

2. $S=$ finite monoid

3. $\left(x \in S, \exists x^{\prime} \in S: x x^{\prime}=1\right) \rightarrow\left(x^{\prime}=\right.$ right inverse of $\left.x\right)$

4. $\left(x \in S, \exists x^{\prime} \in S: x^{\prime} x=1\right) \rightarrow\left(x^{\prime}=\right.$ left inverse of $\left.x\right)$

5. $\left(x \in S, \quad \exists k, \ell \in \mathbb{N}, \quad k<\ell, \quad x^{k}=x^{\ell}\right) \leftrightarrow(x=$ periodic $)$

*All authors with their affiliations appear at the end of this paper.

†Corresponding author: mplobo@uft.edu.br | Join the Open Mathematics Collaboration 


\section{Proposition}

6. An element of a finite monoid is right invertible if and only if it is left invertible. [1]

\section{Proof 1}

7. Proposition: $(S=$ finite monoid $) \rightarrow(S=$ periodic $)$.

8. $(\rightarrow)$

9. Suppose $x \in S$ is right invertible.

10. $\exists x^{\prime} \in S: x x^{\prime}=1$

11. Since $S$ is finite, from (7), $S$ is periodic.

12. From (5) and (11), $x^{k}=x^{k+\ell}=x^{\ell+k}$ for some $k, \ell \in \mathbb{N}$.

13. $1=x x^{\prime}=x^{k} x^{\prime k}=x^{k+\ell} x^{\prime k}$

14. From (12) and (13), $x^{k+\ell} x^{\prime k}=x^{\ell+k} x^{\prime k}=x^{\ell} x^{k} x^{\prime k}=x^{\ell}$

15. Then $1=x^{\ell}=x^{\ell-1} x$.

16. So $x^{\ell-1}$ is a left inverse of $x$.

17. $(\leftarrow)$

18. Similarly, if $x$ is left invertible, it is right invertible.

19. Therefore,

$$
\text { ( } x \in S \text { is right invertible }) \leftrightarrow(x \in S \text { is left invertible }) .
$$




\section{Proof 2}

20. $T(S)=$ transformation semigroup

21. From Cayley's theorem, $S \cong T(S)$.

22. So, the element $x \in S$ corresponds to a transformation $t_{x}: S \rightarrow S$.

23. If $x$ is right invertible, then $x x^{\prime}=1$.

24. Thus $t_{x} t_{x^{\prime}}=t_{1}$.

25. Since $t_{1}$ is a permutation and $S$ is finite, $t_{x}$ is also a permutation.

26. Then, for some $n$, we have $\left(t_{x}\right)^{n}=t_{1}$.

27. In other words, $\left(t_{x}\right)^{n-1}$ is the left-inverse of $t_{x}$, i.e., $x^{n-1}$ is the leftinverse of $x$.

\section{Final Remarks}

28. $S$ = finite monoid

29. ( $x \in S$ is right invertible $) \leftrightarrow(x \in S$ is left invertible $)$

\section{Open Invitation}

Review, add content, and co-author this paper [3,4]. Join the Open Mathematics Collaboration (https://bit.ly/ojmp-slack). Send your contribution to mplobo@uft.edu.br.

\section{Open Science}

The latex file for this paper together with other supplementary files are available [5]. 


\section{Ethical conduct of research}

This original work was pre-registered under the OSF Preprints [6], please cite it accordingly [7]. This will ensure that researches are conducted with integrity and intellectual honesty at all times and by all means.

\section{Acknowledgement}

+ Center for Open Science https://cos.io

+ Open Science Framework https://osf.io

\section{References}

[1] Cain, Alan J. Nine Chapters on the Semigroup Art. AJC Porto \& Lisbon, 2020. http://www-groups.mes.standrews.ac.uk/\%7ealanc/teaching/m431

[2] Lobo, Matheus P. "Open Mathematics Knowledge Base." OSF Preprints, 13 May 2020. https://doi.org/10.31219/osf.io/evq8a

[3] Lobo, Matheus P. "Microarticles." OSF Preprints, 28 Oct. 2019. https://doi.org/10.31219/osf.io/ejrct

[4] Lobo, Matheus P. "Simple Guidelines for Authors: Open Journal of Mathematics and Physics." OSF Preprints, 15 Nov. 2019. https://doi.org/10.31219/osf.io/fk836

[5] Lobo, Matheus P. "Open Journal of Mathematics and Physics (OJMP)." OSF, 21 Apr. 2020. https://doi.org/10.17605/osf.io/6hzyp

[6] COS. Open Science Framework. https://osf.io 
[7] Lobo, Matheus P. "An Element of a Finite Monoid Is Right Invertible If and Only If It Is Left Invertible." OSF Preprints, 28 June 2020. https://doi.org/10.31219/osf.io/7vm92

\section{The Open Mathematics Collaboration}

Matheus Pereira Lobo (lead author, mplobo@uft.edu.br) $)^{1,2}$

https://orcid.org/0000-0003-4554-1372

${ }^{1}$ Federal University of Tocantins (Brazil)

${ }^{2}$ Universidade Aberta (UAb, Portugal) 